\title{
Large-scale flows and convection at the base of solar convection zone
}

\author{
Evgeniy Tikhomolov \\ TRIUMF, Canada's National Laboratory for Nuclear and Particle Physics, \\ 4004 Wesbrook Mall, Vancouver, BC, V6T 2A3, Canada \\ email: etikhomolov@solar.stanford.edu
}

\begin{abstract}
Development of convection in sun's outer shell is caused by reduction of effectiveness of energy transfer by radiation. Traditionally, models of solar convection are considered to be axisymmetric on the scale of solar radius. Such models provide basic understanding of convection under solar conditions. However, interpretation of a number of observable large-scale long-lived solar phenomena requires developing a non-axisymmetric approach. We present such a model in which large-scale non-axisymmetry is caused by large-scale flows such as Rossby waves and vortices. We model flows near the base of the solar convection zone. Anelastic approximation is used, which is valid for flow velocities much smaller than local sound speed. Our three-dimensional numerical simulations show that interaction of convection with large-scale flows leads to the establishment of non-axisymmetric large-scale temperature distribution. The interaction also gives rise to large-scale variations of penetration depth of convective plumes. Generation of the magnetic field by large-scale non-axisymmetric flows can explain such solar phenomena as complexes of activity, active longitudes, drifts of large-scale magnetic fields from equator to the poles, and appearance of distinct rotation periods of magnetic fields at some latitudes. We discuss a possibility of detection of large-scale non-axisymmetric flows and temperature distributions associated with them by the methods of helioseismology.
\end{abstract}

Keywords. Convection, instabilities, radiative transfer, turbulence, Sun: rotation

\section{Introduction}

It is a well-known fact that the sources (or amplifiers) of solar magnetic fields are hydrodynamic flows. But, if we go into details to answer the question "What are the sources of long-lived large-scale magnetic structures (LSMS) and associated with them complexes of activity?", the situation is less definite. By the term "large-scale" we mean more than ten heliodegrees in latitude and longitude. The diffusion time of such structures can be evaluated as $L^{2} / D$, where spatial scale is on the order of $L \sim 10^{10} \mathrm{~cm}$ and effective turbulent diffusion is evaluated as $D \sim 10^{13} \mathrm{~cm}^{2} / \mathrm{s}$. It gives a life-time on the order of 4 month. This estimation is in good correspondence with a life-time of separate complexes of activity. However, new activity regions appear in vicinity of the old, decayed one. Thus a recurrence of activity can be observed in the same area for much longer time. The extreme manifestations of such recurrences are the active longitudes which can live up to several years. Obviously, such long-lived phenomena can't be explained by random excitation of magnetic structures in the convection zone and their subsequent decay.

Initially, in 1970th, the LSMS were explained as non-axisymmetric modes in a dynamomechanism (Stix (1971)). Then, Mogilevsky et al. (1986) suggested that LSMS are formed as a result of conglomeration of interacting small-scale magnetic elements. Such models are being developed up to date using technique of cellular automata (Vlahos et al. (2002)). And not so long time ago we developed a model in which LSMS are generated by large-scale vortical flows (LSMF) (Tikhomolov 1995). Thus, in our approach, the 


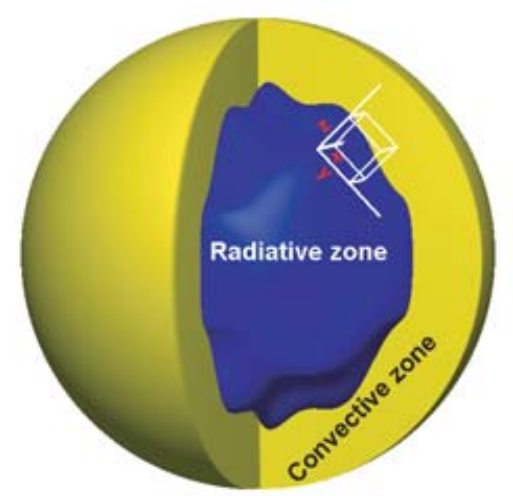

Figure 1. Modelling region near the deformable interface between the convective and radiative zones

question about the sources of LSMS is transformed into a question about the sources of LSVF. In this sense, our approach can be called hydrodynamic. Why do we need another alternative hydrodynamic theory for the generation of LSMS? The answer is that it promises to be the common basis for explanation of many observable phenomena such as distinct rotation periods, vortical distribution of magnetic field, north-south asymmetry in rotation (Tikhomolov 1998), and even 11-year solar cyclicity (Tikhomolov 2001). Now it is too early to talk about the "unified hydrodynamic theory" for large-scale long-lived solar phenomena but first results in that direction are encouraging.

Diffusion time evaluated for LSMS in the convection zone can also be applied to LSVF. This diffusion time is too small to explain the long-lived large-scale structures by excitation of LSVF in the convection zone or on the solar surface. Thus, we give preference to excitation of LSVF at the bottom of the solar convection zone, more exactly in the penetrative convection region. In that region, the effective turbulent viscosity has a value much smaller than in the convection zone. LSVF can be excited by turbulent convection itself in random manner. However, the behavior of the LSVF traced by magnetic field shows that a random excitation and a subsequent decay (now it is very long!) is not enough to explain the behavior of LSMS. It would be preferable if some other mechanism would exist, which is able to sustain LSVF. In this paper we discuss such a mechanism.

\section{The model}

Convection in the Sun develops in the region where heat transfer by radiation becomes ineffective. The location of critical depth at which fluid starts to move is determined by many factors, but the main factor is the temperature decrease (and a corresponding increase of opacity). Traditionally, models of solar convection are considered to be axisymmetric on the scale of solar radius; the critical depth is constant. Such models provide basic understanding of convection under solar conditions. However, large-scale flows change the situation dramatically: they introduce temperature and density disturbances which lead to a deformation of the interface. This deformation leads to a strong interaction between large-scale flows and a small-scale convection at the base of the solar convection zone.

In this paper we present a model in which the critical depth for convection instability is dependent on local temperature: the deformed interface follows the temperature 
disturbances. Deformation of a critical surface is schematically shown in Figure 1 (it is exaggerated on this figure!).

We consider a region of rotating spherical fluid layer in Cartesian coordinates. The hydrodynamical flows are modelled in the anelastic approximation. It means that sound waves are filtered out. It is a legitimate approximation for flow velocities much smaller than sound speed. Physical parameters for the base of the solar convection zone are taken from the standard solar models. The dimensionless equations for the velocity components $V_{i}$, pressure $P$, temperature $T$, and density $\rho$ are represented in tensor notations:

$$
\begin{gathered}
\partial_{0}\left(\rho V_{i}\right)+\frac{1}{P r} \operatorname{div}_{j}\left(\rho V_{i} V_{j}\right)=-\partial_{i} P+\operatorname{div}_{j}\left(\tau_{i j}\right)+\frac{1}{P r F r^{2}} \rho n_{i}+\frac{1}{E k} \rho \epsilon_{i j k} \omega_{j} V_{k} \\
\partial_{0}(\rho T)+\frac{\gamma}{\operatorname{Pr}} \operatorname{div}_{i}\left(\rho T V_{i}\right)=\frac{\gamma E c}{\operatorname{PrFr}^{2}} \rho n_{i} V_{i}+\frac{\gamma}{\operatorname{Pr}} \operatorname{div}_{i}\left(K \partial_{i} T\right)+\gamma E c \operatorname{div}_{i}\left(\tau_{i j} V_{j}\right) \\
\operatorname{div}_{i}\left(\rho V_{i}\right)=0 \\
\rho=\operatorname{PrEc} \frac{\gamma}{(\gamma-1)} \frac{P}{T}
\end{gathered}
$$

where

$$
F r^{2}=\frac{\chi_{0}^{2}}{g H^{3}}, E c=\frac{\chi_{0}^{2}}{c_{p} T_{0} H^{2}}, \operatorname{Pr}=\frac{\nu_{0}}{\chi_{0}}, E k=\frac{\nu_{0}}{2 \Omega_{0} H^{2}}
$$

are squared Froude, Eckert, Prandtl, and Ekman numbers respectively.

Boundary conditions are: at the top and bottom the temperature is constant and the upper and lower surfaces are impenetrable and stress-free. In longitude (axis x) and latitude (axis y) periodic boundary conditions are implied. The region is divided into two parts. In a static case, upper one-third and lower two thirds are, respectively, convectively unstable and stable. The dominance of radiative transfer in the lower layer is modelled by increasing the thermal diffusivity by 10 times with respect to its value in the upper layer. When convection begins, the location of the surface dividing convectively stable and unstable parts depends on local temperature and density.

We take the first linear terms in dependence of surface disturbance $h(x, y)$ on disturbance of temperature and density:

$$
h(x, y)=c_{1} \delta T+c_{2} \delta \rho,
$$

where $c_{1}$ and $c_{2}$ are free parameters of the problem. In this paper, we investigate only the influence of temperature on the location of the interface. Thus $c_{2}=0$.

\section{Results}

Deformable interface between convectively stable and unstable regions adds new features to the flow pattern. Figure 2 shows convection in a turbulent regime for a case without rotation. Different level of transparency shows the deviation of temperature from the mean value at each depth. Velocity field is shown by cones. The interface between the convectively-stable and unstable parts of the layer is represented by the surface which looks like a net. The deformation of the interface is determined by the temperature disturbances. In cool regions with downflows, the interface is deflected in the bottom direction. For warmer regions, the case is opposite. Different boundary conditions on the top and bottom of the convectively-unstable layer create asymmetry between the up- and downflows. The flow pattern changes dramatically without hard lower boundary: downflows concentrate into narrow plumes and penetrate to some depth where they are decelerated 


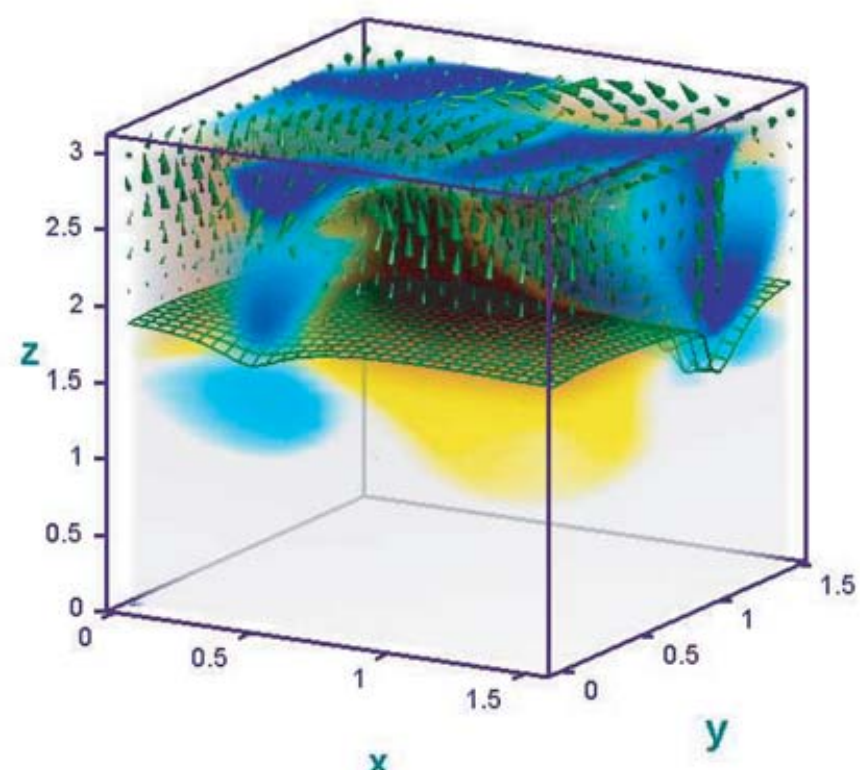

Figure 2. 3D numerical simulations of convection in the region with deformable interface without rotation.

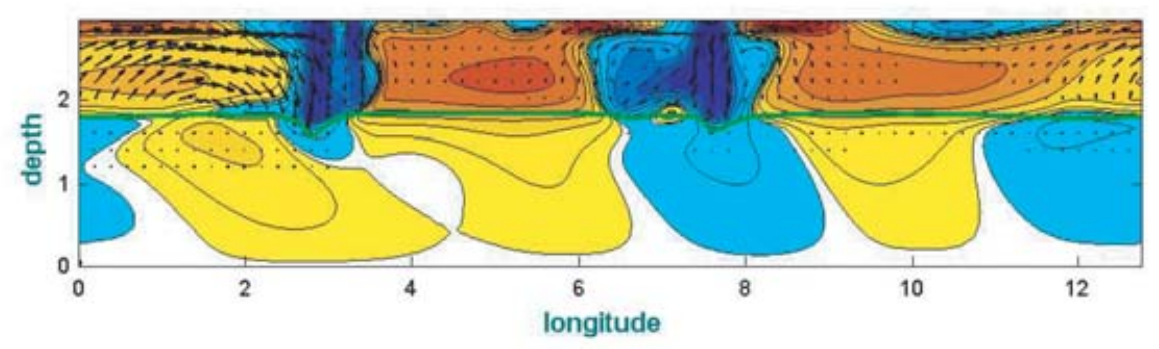

Figure 3. 2D numerical simulations of convection in the region with deformable interface without rotation.

and loose their momentum. For our case of deformable interface, an additional asymmetry appears. Because the thickness of the convectively-unstable layer is larger in cooler regions, the downflows have larger velocities. Thus, penetration depth of the plumes is larger. The behaviors of small-scale convection and large-scale flow component are simulated in two dimensions. Figure 3 shows stationary convection without rotation and the large-scale component. The distribution of temperature disturbances are shown by solid contours and filling. Velocity field is represented by arrows. The deformed interface is represented by a wide solid line. One can see that downflows are concentrated in narrow plumes. Upflows are much wider. For better presentation, the velocity field is cut-off at 


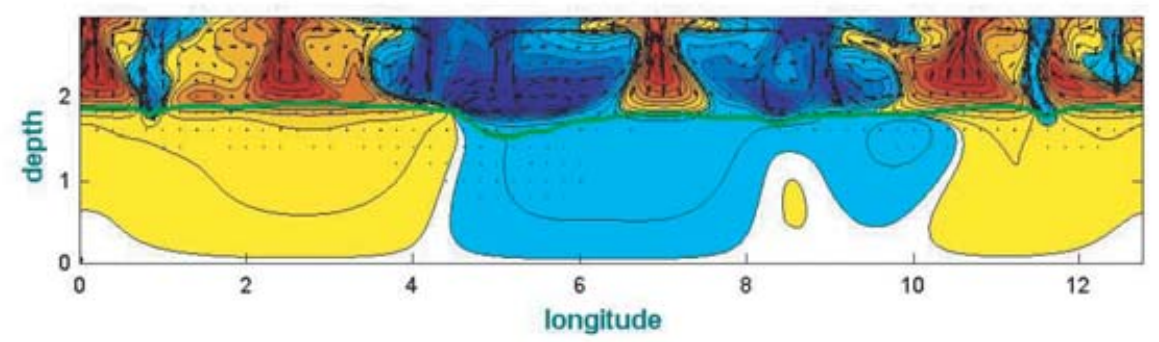

Figure 4. 2D numerical simulations of convection in the region with deformable interface with rotation and the large-scale component.

the level of five percent of its maximum value. Such value of velocity is observable down to half of convectively-unstable layer thickness.

An interesting feature of the solution is a particle-like behavior of one convective cell. It moves from left-hand side of the figure to the right. On its way, it absorbs other cells, but keeps its identity for a very long time. We can't associate this behavior with any observable phenomena known at present. For us in this paper, this is a "side-effect" which appears unexpectedly and, probably, deserves an investigation in a separate work.

Introducing rotation adds additional features to the convection pattern: a strong horizontal vortical component appears, convective motions are suppressed by rotation, and the scale of convective cells decreases. Solar conditions correspond to the situation when rotation significantly influences large-scale flows but not small-scale convection. We model this situation by appropriate choosing of the dimensionless parameters. After the establishment of a stationary regime for rotation case, a large-scale disturbance was added to the temperature field. Then we observe how the system responds to this change. The addition of large-scale temperature component leads to a large-scale deformation of the interface and a subsequent modulation of convection: in the areas of deflection, convection is intensified, and vice versa. Convective cells appear and disappear in a random manner. Modulated convection itself creates a large-scale temperature component. Thus we have positive feedback in a chain: large-scale temperature distribution $\rightarrow$ large-scale deformation of the interface $\rightarrow$ modulated convection. This feedback leads to the establishment of a long-lived large-scale structure which combines small-scale convection, deformed interface, and a large-scale temperature distribution. This structure is shown in Figure 4. Potentially, such long-lived large-scale hydrodynamic structures can be the sources of large-scale long-lived magnetic structures visible on the solar surface.

\section{Prospects}

We found that due to deformation of the interface between convectively-stable and unstable layers, large-scale vortical structures can be sustained for a very long time, and potentially can be the sources of large-scale magnetic structures and associated with them complexes of activity. Probably, a helioseismic time-distance technique (Zhao \& Kosovichev (2003)) can be applied for detection of LSVF. Stereo view of the solar surface can also help to identify LSVF. Note that if only the velocity perturbations would exist, the detection of LSVF would be very difficult because of strong noise from convection. But along with the velocity perturbations, the large-scale temperature variations 
appear, which are associated with the deformation of the interface between convectively stable and unstable layers. Therefore, sound speed varies in accordance with large-scale temperature perturbations. It seems that it this case the detection of LSVF is more probable.

\section{References}

Antonucci, E., Hoeksema, J.T. \& Scherrer, P.H. 1990, ApJ 360, 296

Bumba, V. 1987, Bull. Astron. Inst. Czech 38, 92

Mogilevsky, E.I. et al. 1986, in: A. Antalova (ed.), Contr. Astron. Obs. Skalnate Pleso, Proc. 12th Region. Consult. on Solar Phys., Astron. Ustav Slovenskej Akad. Vied (Tatranska Lomnica), vol. 15 , p. 189

Stix, M. 1971, A\& A 13, 203

Vlahos, L., Fragos, T., Isliker, H. \& Georgoulis, M. 2002, ApJ 575, L87

Tikhomolov, E. 1995, Sol. Phys. 156, 205

Tikhomolov, E. 1998, Astron. Lett. 24234

Tikhomolov, E. 2001, Solar Phys. 199165

Zhao, J. \& Kosovichev, A.G. 2003, ApJ 591, 446

\section{Discussion}

KUPKA: Did you consider to use your numerical modelling of the interface between the stable and the unstable zone to derive some scaling laws the stellar evolution community could use to describe mixing in the overshooting layers?

Tikhomolov: Yes, it could be done. But we have only run this for parameters related to the Sun.

BRUMmell: How do your anelastic simulations compare to the $2 \mathrm{D}$ and $3 \mathrm{D}$ compressible simulations that have been performed earlier on exactly the same subject?

TikHomolov: I don't know the works which model large-scale flows and small-scale convection at the same time at the base of the solar convection zone with fully compressible code. For such flows, fully compressible simulations should give exactly the same results as in anelastic approximation, but they are more complicated and time-consuming.

BrAndenBurg: How the large-scale component is sustained in your numerical simulations?

Tikhomolov: When interface is deformed, small-scale convection is modulated on the large-scale and itself gives large-scale temperature gradients. These temperature gradients lead to the appearance of a large-scale velocity field. Thus we have positive feedback between a small-scale convection and large-scale flows provided by the deformable interface.

RoxBurgh: Concerning the previous question, what is clear in your work is the fact that you get large scale inhomogeneities. My question: do these inhomogeneities also emerge automatically? It seemed that you put them in initially.

Tiknomolov: The temperature disturbances are added to the established, stationary temperature field. Then we look how the system responds to these disturbances. For the rotating case the disturbances are amplified. 